\title{
Cognitive Radio Ad-Hoc Networks: Some New Results on Multi-Channel Hidden Terminal Problem
}

\author{
Palaniandavar Venkateswaran ${ }^{1}$, Srishti Shaw ${ }^{1}$, Sandhya Pattanayak ${ }^{2}$, Rabindranath Nandi ${ }^{1}$ \\ ${ }^{1}$ Department of Electronics \& Telecommunication Engineering, Jadavpur University, Kolkata, India \\ ${ }^{2}$ Department of Electronics \& Communication Engineering, Narula Institute of Technology, Kolkata, India \\ Email:pvwn@ieee.org, srishti67@gmail.com,sandhya.pattanayak@yahoo.com,robnon@ieee.org
}

Received September 17, 2012; revised October 15, 2012; accepted November 15, 2012

\begin{abstract}
Recently the Cognitive Radio (CR), in particular the CR Ad-Hoc Network (CRAHN) technology appears as a burgeoning area in wireless communication that enables utilization of limited network resources in more efficient and intelligent way; studies indicate that opportunistic utilization of the available radio frequency spectrum, without interfering the licensed primary user (PU) could be made. This paper presents some simulation based performance of the MultiChannel Hidden Terminal (MCHT) problem on CRAHNs; new observations on the effect of the number of channels on certain PU-activity metrics, e.g., delay and throughput, are described.
\end{abstract}

Keywords: Cognitive Ad-Hoc Networks; MCHT Problem; Wireless Communication; NS-2

\section{Introduction}

Recent research studies indicate that wireless data traffic would grow@90\% while having to handle hundreds of petabytes in this decade [1]; with such an enormous mobile data usage, spectrum crunch is inevitable. Currently the $\mathrm{CR}$ technology is considered to be the practicable solution to address the situation. Most of the bands allocated to licensed PUs remain unutilized at a given geographic location at certain time slots [2,3]. The evolution of self organizing infrastructure less mobile ad-hoc networks [4] may be combined with the CR technology with a view to configuring new CRAHN for intelligent and opportunistic use of unutilized bands for the Secondary User (SU) without interrupting the PU activity.

The CR technology is now going through a phase of active research and development activity with several challenges towards spectrum sensing/allocation and on the MCHT problem [5-10]. Various MAC protocols were proposed for a solution to the hidden terminal problem in ad-hoc networks. The dynamic nature and the multiple number of channels make the medium access more challenging. The performance of certain metrics e.g., throughput, packet delivery ratio etc. is expected to be better with respect to that of single channel owing to reduction in interference [10]; but here presence of unidentified hidden terminals tries to limit the performance of CRAHNs $[11,12]$.

We observe from the literature $[7,8]$ that exploiting multiple channels, one obtains higher throughput than using one channel since multiple transmission may be obtainable without interference. In [8], the transmission characteristics had been studied for various protocols taking the average throughput and packet delay as the two metrics. This study indicates that the single-channel Distributed Function Protocol (802.11 DCF) does not work well in a multi channel environment since it is designed for sharing single channel between hosts. Thus when a host is listening to a particular channel, it is unable to communicate on another channel. This gives rise to the MCHT problem. Under these practical considerations we investigated the MCHT problem with the simulation scenario taking into account another set of metrics.

In this paper, we describe the MCHT in context of CRAHNs and present a simulation based study to provide an insight as to how the different network parameters (number of channels, number of PUs, etc.) affects the network performance under MCHT scenario. The CRAHNs is a more practicable solution than their centralized counterparts, due to the rapid deployability, decentralized and self-organizing nature of such networks. However, it offers more research challenges due to the absence of any central control agent. The paper is organized as follows: in Section 2, aspects of the MCHT problem and how it affects the performance of CRAHNs are described; Section 3 lists a literature survey and Section 4 presents a comprehensive NS-2 based simulation study on the characteristic features of MCHT on CRAHNs. In Section 5 we interpret the findings with some concluding remarks. 


\section{MCHT Problem for CRAHNs}

Some basic outlines of the MCHT problem for CRAHNs are discussed by using the schematic diagrams in Figures 1 and 2, assuming a multi-channel CR-MAC protocol that does not address this problem. The hidden terminal problem occurs when two terminals say $\mathrm{A}$ and $\mathrm{C}$, in Figure 1, are within the range of the station B but out of the range of each other, or separated by some physical obstacles transparent to radio signals. These two terminals are said to be hidden from each other resulting in interference [7] at B. It is improved by the four-way handshaking methodology [13] in which a control signal in form of a short message is sent first and then the data packets are transmitted only when the receiver permits. Therefore the collision only happens during the transmission of the request signal but not in that of the traffic signal. In this way, the hidden terminal problem is alleviated, as in IEEE 802.11 MAC.

However, hidden terminal problem and MCHT issue [8] are more complex to CRNs. Hereafter, we postulate a new type of hidden terminal problem pertaining to CRNs and name it as the cognitive radio multi-channel hidden terminal problem (CR-MCHT). The CR-MAC assumed is similar to [14] but it has only one transceiver. Assume $\mathrm{N}$ channels are available; one channel is dedicated for exchanging control messages (control channel), and all the others are for data exchange. When a node is neither transmitting nor receiving, it listens to the control chan- nel. The assumed CR-MAC supports the PU activity. If any $\mathrm{CR}$ user (SU) is transmitting on a channel and PU wants access to it, the CR-MAC commands the SU to immediately vacate the channel and continue its transmission on an another vacant channel, if available or wait for the PU to complete its transmission/reception. We observe that the CR-MCHT problem can cause two types of interference: interference caused at PU by a hidden $C R$ and interference caused at a CR by another hidden CR.

\subsection{Interference at $P U$}

When node A wants to transmit a packet to node $\mathrm{B}, \mathrm{A}$ and $\mathrm{B}$ reserve the channel by exchanging "Request to Send" (RTS) and "Clear to Send" (CTS) messages. When sending an RTS, node A includes a list of channels it is trying to use. On receiving the RTS, B selects a channel and includes the selected channel in the CTS. Subse-

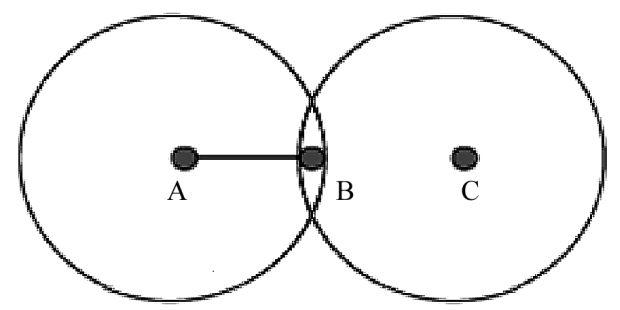

Figure 1. Nodes $A$ and $C$ are hidden with respect to each other.

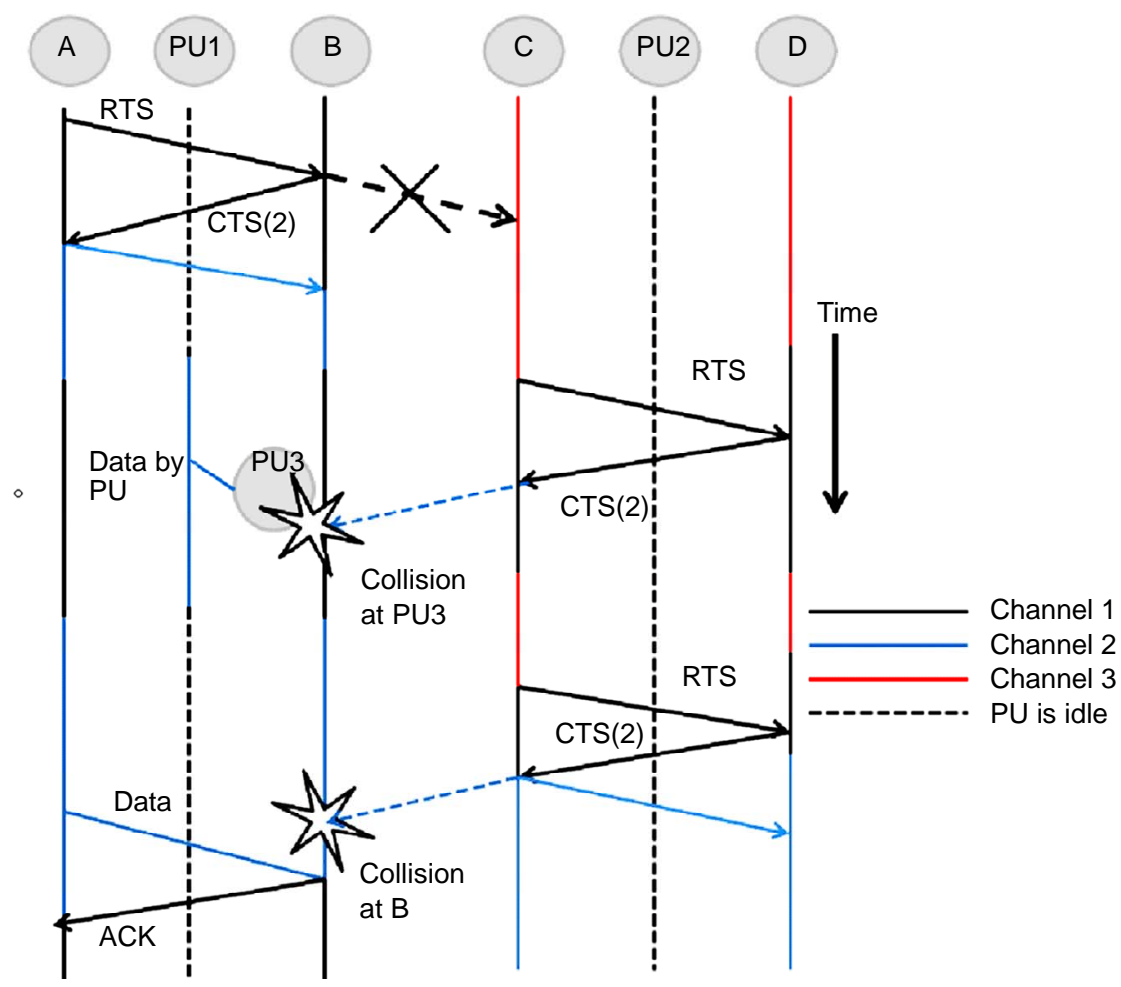

Figure 2. Multi-channel hidden terminal problem. 
quently, node $\mathrm{A}$ and $\mathrm{B}$ switch their channels to the agreed data channel and exchange the DATA and ACK packets. When this four-way handshake completes, nodes A and $\mathrm{B}$ immediately switch to the control channel. In the given scenario when $\mathrm{A}$ has a packet for $\mathrm{B}, \mathrm{A}$ sends a RTS on Channel 1, which is the control channel. B selects Channel 2 for data communication and sends a CTS back to A. The RTS and CTS messages reserve Channel 2 in the transmission ranges of $\mathrm{A}$ and $\mathrm{B}$; no collision occurs. During this period the Primary user 1 (PU1) is either not present or not transmitting; at a subsequent time slot, even though the transmission of $\mathrm{A}$ and $\mathrm{B}$ are not complete, PU1 demands access to Channel 2; note that C and $\mathrm{D}$ also are using Channel 3. Thus as no channel is empty, A and B has to withhold their data transmission for some time.

The PU1 starts transmitting data to PU3 on Channel 2; but, during this time $\mathrm{C}$ and $\mathrm{D}$ were busy exchanging data on Channel 3. Unaware of the fact that PU3 is receiving on Channel 2, node $\mathrm{C}$ initiates a communication with Node D, and ends up selecting Channel 2 for communication. This results in interference or collision at PU3.

\subsection{Interference at Another $\mathbf{C R}$}

Referring to Figure 2 again one observes that collision at PU3 causes $\mathrm{C}$ to defer its transmission by an abrupt period of time, decided by the back-off timer. After normal RTS-CTS exchange (not shown in Figure 2), C and D starts communicating on Channel 3. By this time PU1 and PU3 have completed their data-exchange and nodes $\mathrm{A}$ and $\mathrm{B}$ have resumed their transmission. Node $\mathrm{C}$ once again initiates a communication with $\mathrm{D}$ and selects Channel 2, not knowing the on-going transmission between A and B on Channel 2. Thus a collision occurs at B.

The above problem occurs due to the fact that nodes may listen to different channels, which makes it difficult to use virtual carrier sensing to avoid the hidden terminal problem. If each node listens to only one channel, node $\mathrm{C}$ would have heard the CTS and thus deferred its transmission. Thus the normal four-way hand shaking (RTSCTS-DATA-ACK) cannot address the CR-MCHT problem, where the presence of PUs and multiple SUs makes the problem somewhat complex.

\section{Literature}

To enhance the performance of ad-hoc networks the concept of cognitive radio can be infused into it so as to make an Opportunistic Spectrum Access [12]. The major challenges faced are the MCHT problem and multi-channel unreachability problems leading to unreliable local broadcast in mobile ad-hoc networks. RTS/CTS handshake technique was suggested for preventing hidden terminal problem and is dependent on the transmission range (Rtx), carrier sensing range (Rcs) and interference range (Ri). In [15], it is studied that the effectiveness of RTS/CTS process reduces when the value of $d$ exceeds $0.56 *$ Rtx , where $d$ is the distance between the transmitter and receiver and Rtx being the transmission range [13]. Some cognitive ad-hoc protocols designed to solve these problems [15-18] suggest consideration for dynamic channel selection without any centralized coordination [19]. The analysis of hidden terminal problem has been done in [20-22]. In [20], it has been found that the hidden node problem will increase the multiple retries by nodes, making wrong declaration of link failures leading to re-routing instability. Due to the hidden nodes vulnerable period occurs leading to collision of packets. This probability of collision during transmission may be due to ACK-ACK collision or DATA-DATA collision. It is seen that the maximum sustain-able throughput is limited by the vulnerable periods which depend on the numbers of hidden nodes and the fraction of airtime in the time horizon when hidden-node collisions may occur [20]. Other work related to SNR detection, probabilistic switch policy and application of ANN [23-25] have also been recently reported. A comprehensive and current survey on the topic may also be consulted in $[5,6,26,27]$.

\section{Proposed Study}

In this section, we describe the simulation scenario using two metrics to ascertain the performance of the CRN under CR-MCHT problem:

a) Network Throughput: Use of multiple channels is expected to increase the network throughput. Ideally the improvement factor must be a factor of $\mathrm{N}$ for $\mathrm{N}$-channel system. But in practice, this is not possible due to the overhead involved in channel negotiation and frequent disruption caused in communication by the PUs.

b) End-to-End Packet Delay in the network: End-to-end packet delay, or simply delay, is the duration between the time when the Link layer of the sender receives a packet to send, and the time the packet reaches the destination. So the delay is the sum of delays for queuing, backoff, channel negotiation and transmission delay.

\subsection{Description of Scenario}

The proposed investigation scenario is shown in Figure 3. The distance from node 1 to node 2 and node 3 to node 4 is fixed at $250 \mathrm{~m}$. Transmission range of the wireless radio is $367 \mathrm{~m}$. The channel bandwidth is variable depending on the channel used. We use three channels bandwidths: $2 \mathrm{Mbps}$, $5.5 \mathrm{Mbps}$ and $11 \mathrm{Mbps}$, following the 802.11 standard. We vary the vertical distance be- 
tween nodes 2 and 3 to check the influence of large interference range. Two CBR sessions based on UDP are involved with directions from node 1 to node 2 and node 4 to node 3 correspondingly. Since the CBR is constant rate traffic without re-transmissions, it is possible that the two flows may synchronize to each other rendering the results not general enough. To avoid the synchronization of the two flows, we slightly modified the CBR traffic generator. Given the rate as n packets per second (pps), we divided time into slots as $1 / \mathrm{n}$ seconds. In each time slot, a packet is sent to the network. Sending time of the packet is uniformly distributed in the whole slot. We varied three parameters of the scenario: distance, packet rate (pps) and number of PUs. Table 1 shows the common parameters for the three scenarios.

It may be added here that the IEEE 802.11 standard for wireless LAN [28,29] provides multiple channels for practical simulation work; IEEE 802.11 b physical layer (PHY) has 14 channels with $5 \mathrm{MHz}$ frequency separation, wherein for the bands to be non-overlapping, the separation space is specified at $30 \mathrm{MHz}$. Channels 1, 6 and 11 are typically used for such communication.

These numerical standards had been followed as per compatibility with $[7,8,28]$. The results obtained in the proposed work, albeit viewing the problem with a different set of metrics, are found to be comparable as those in [8].

The scenario discussed here for the proposed work had been created intuitively after a thorough study of literatures cited in $[7,8,13,29]$ where metrics were selected by these authors of their own choice. We noted that there are relatively fewer references related to the MCHT problem and we selected our studies towards transmission characteristics under the MCHT problem selecting our own metrics (e.g., delay, throughput).Under either approach, interestingly, results were found to be fairly concomitant. The work in [29] had been carefully studied and adopted as the theoretical basis of the present work.

\subsection{Simulation Model}

The cognitive radio model in [11] had been used for the

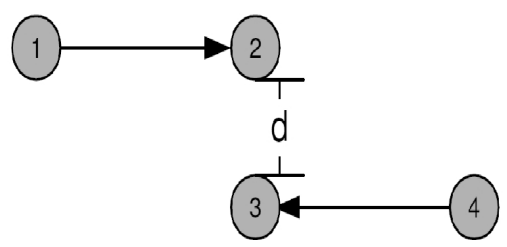

Figure 3. Investigation scenario.

Table 1. Parameter settings for the environment.

\begin{tabular}{cc}
\hline Area of simulation & $500 \times 500 \mathrm{~m}^{2}$ \\
\hline Simulation time & 100 seconds \\
Number of channels & $3,7 \& 11$ \\
\hline
\end{tabular}

simulation purpose. This model enables the PU and CR user activity to be simulated and the radio interfaces are equipped with cognitive radio capabilities. This model uses two policies for switching the channel of CR user when PU activity is detected on the current channel: Probabilistic switch and Always switch. Section 2 describes the CR-MCHT problem using the always switch policy, which causes $\mathrm{CR}$ to vacate the channel immediately when PU activity is detected. According to the probabilistic switch policy, the $\mathrm{CR}$ vacates the channel when the switching probability increases above a "Switch-threshold". This value depends on the interference level allowed at the PU $[23,24]$ and hence is a system dependent parameter. For the proposed simulation work, we selected the always-switch policy.

\subsection{Simulation Result}

The simulation had been carried out in our lab and the results are presented in this section. The IEEE 802.11 MAC with the cognitive radio model described in Section 4.2 with support for three to eleven channels is being used. The results corresponding to variations in distance $(d)$, packet rate and number of PUs are presented below in Figures 4-9.

- Varying distance " $d$ ": Figures $\mathbf{4}$ and $\mathbf{5}$ show the simulation results where it has been assumed that a maximum of ten primary users can be present at any instance in course of the simulation time.

When the distance $d$ is low and within the interference range of the nodes, the hidden terminal problem is critical and so the network throughput is low while the packet delay is high. As the distance increases, both the metrics show improvement, i.e., network throughput increases and the packet delay falls. When the number of channels is low (three), Figure 4 shows abrupt increase in throughput and abrupt decrease in delays in Figure 5.

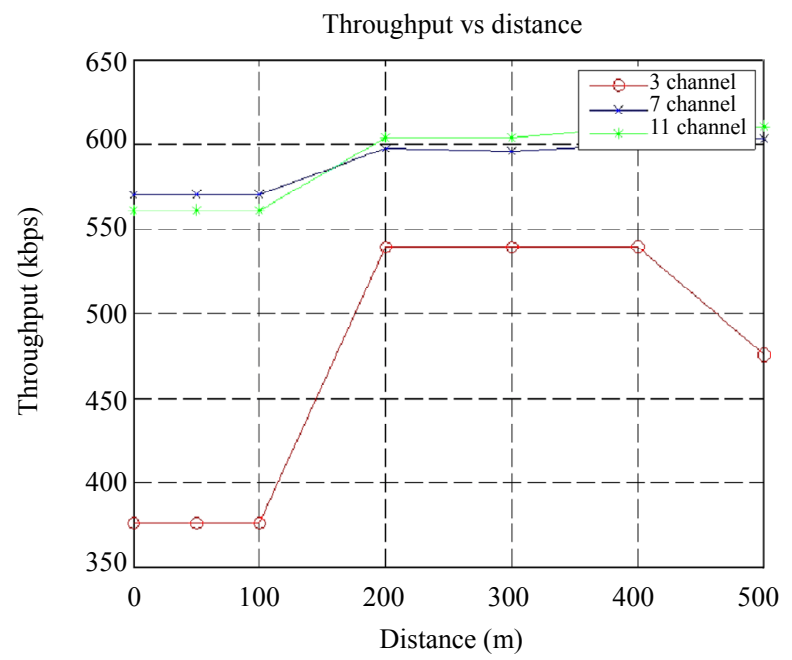

Figure 4. Throughput vs distance. 


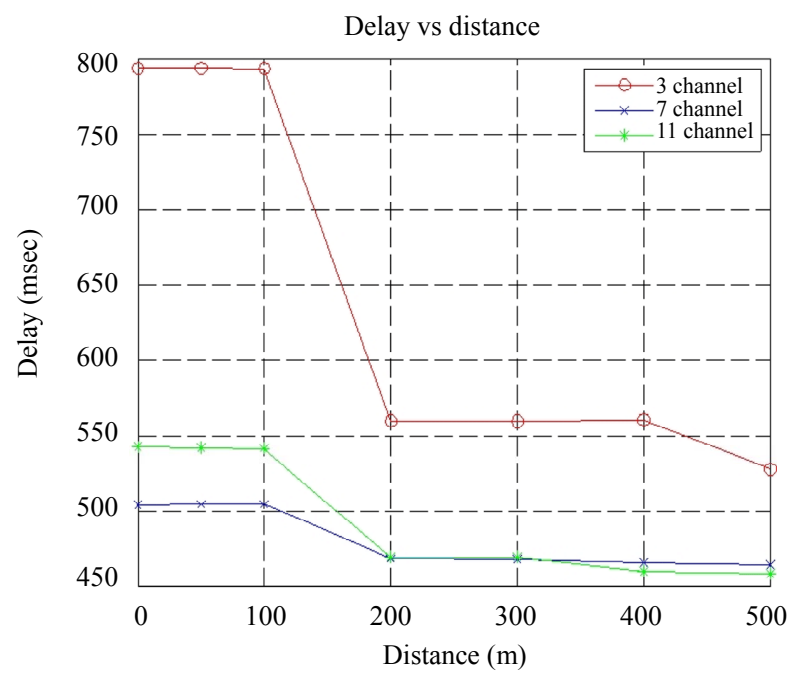

Figure 5. Delay vs distance.

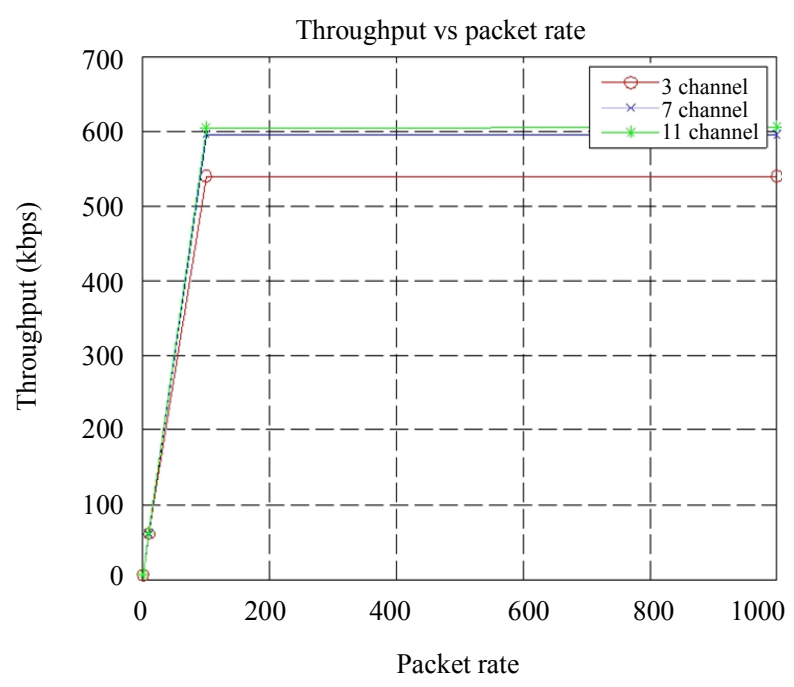

Figure 6. Throughput vs packet rate.

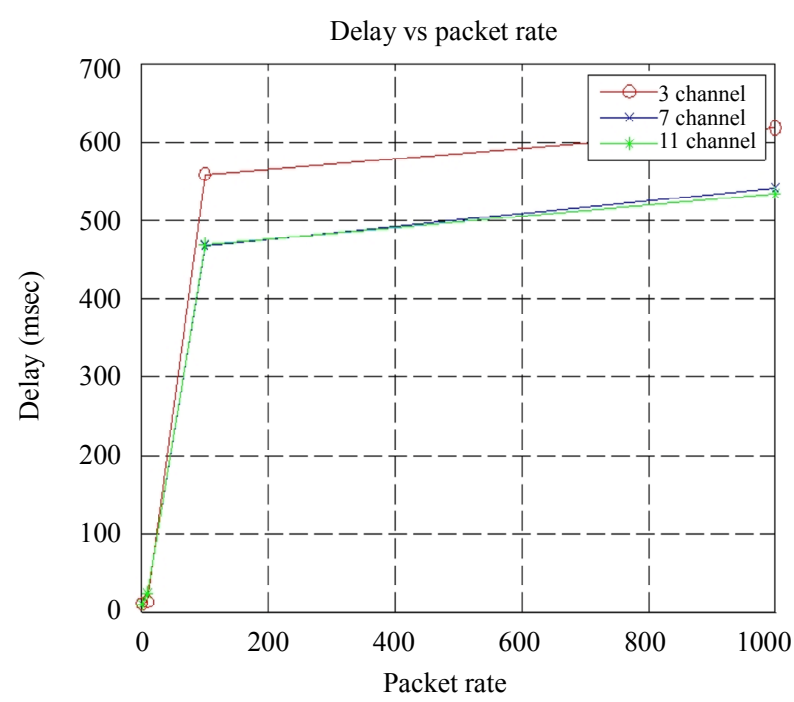

Figure 7. Delay vs packet rate.

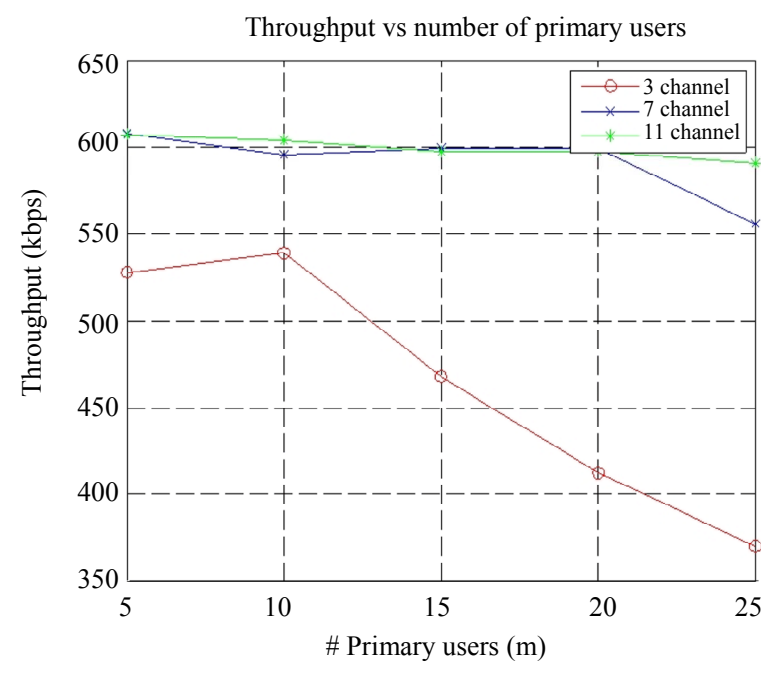

Figure 8. Throughput vs $n$.

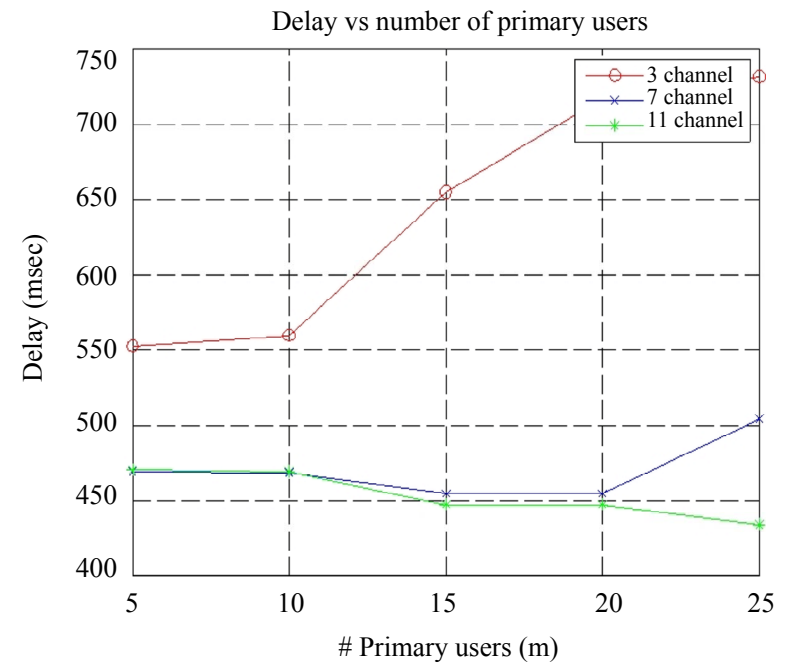

Figure 9. Delay vs $n$.

With increasing number of Channels to 7 and 11 the network shows a nominal improvement. This is because, when the number of channels is less, the CR user has to vacate the channel for PUs and wait for PUs to finish their transmission resulting to throughput improvement. It is noted that when more number of channels are present, the CR users can switch their transmission to an alternative empty channel, which in turn decreases the delay. We observed that too many channels (eleven in our simulation) do not improve the performance. However we obtained some improved result if the number of channel is seven. Albeit the metrics do not show significant improvement for eleven channels, the metric values remain at satisfactory tolerable limits.

Thus, simulation results indicate that an optimum number of channels would give the best solution in terms of throughput and delay of the network. The number is found to be seven in the present study for the given sce- 
nario. In view of obtaining the optimum number of channels for more satisfactory performance, we are now working on a detailed simulation study involving more number of metrics.

- Varying the packet rate: Here distance $(d)$ had been set to $300 \mathrm{~m}$ and number of PUs is fixed at 10; Figures 6 and 7 show the results.

With the increase of number of packets per second, the results indicate that for any number of channels, the throughput increases, but when the packet rate is high ( $\geq 500$ packet/sec.) in Figure 6, there is no improvement in throughput. This is because for high packet rate the packet drop also becomes high. Moreover, the finite channel switching delay becomes very prominent leading to high packet loss. Thus, in such a situation even large number of channels does not seem to improve the performance. The network delay increases almost linearly with the increase in packet rate as in Figure 7; our observation for the reason is owing to increase in network congestion, control channel bottleneck, finite buffer size, etc.

- Varying the number $(n)$ of PUs: We varied the number of PUs keeping the distance $(d)$ fixed at $300 \mathrm{~m}$ and packet rate being set at 100 packets/sec. The observations are shown in Figures 8 and 9.

When three channels are used the network performance shows a marked difference from that when large number of channels are allowed. We observed that if the number of channels is three and number of PUs increases, the throughput increases slightly; the reason being better utilization of channels; but as the number of PUs increases, the throughput falls rapidly. The increasing frequency of channel switching leads to high packet loss, which in turn causes a low throughput.

Similarly high channel switching frequency and finite channel switching time, combined with the contention for the common control channel causes a rapid rise in delay with increasing number of PUs. The results however show a different trend for large number of channels. With increase in the number of the PUs, the throughput and delay remains almost same for large number of channels. Availability of channels allows an alternate medium (channel) for transmission. Also, the performance do not show significant improvement when the number of channels is very high $(n=11$ in this case).

\section{Conclusion}

In this paper, we present some preliminary investigations on MCHT problem on the performance of CRAHNs. It has been suggested that the MCHT problem may be mitigated by some collaborative context using data from distributed spectrum sensors [20]; here we consider a similar context involving the PUs in terms of switching policies. It is found that the full potential of CRAHNs is yet to be realized in presence of the MCHT problem. We varied three parameters of the proposed simulation scenario: distance, packet rate (pps) and number ( $n$ ) of PUs. Albeit, we observed some improvements on the CRAHN characteristics within some range of the metric variation, more investigations would be needed through certain extension of the proposed simulation scenario. Our investigations also indicate that satisfactory metric utilization it may be possible to derive an optimum number of channels. The work presented here is being carried out further in this direction in view of developing a protocol to address the problem.

\section{REFERENCES}

[1] J. Mitola and G. Q. Maguire, "Cognitive Radio: Making Software Radios More Personal," IEEE Personal Communications, 1999, pp. 13-18. doi:10.1109/98.788210

[2] S. Haykin, "Cognitive Radio: Brain-Empowered Wireless Communication," IEEE Journal on Selected Areas in Communications, Vol. 23, 2005, pp. 201-220. doi:10.1109/JSAC.2004.839380

[3] D. Saha and V. Sridhar, "Emerging Areas of Research in Business Data Communication," IEEE Journal on Selected Areas in Communications, Vol. 7, No. 4, 2011, pp. 52-59.

[4] P. Venkateswaran, "Studies on a Class of Mobile Ad-Hoc Networks (MANETs) with Efficient Routing, Mobility/ Interface Modeling \& Improved Clustering Schemes," Ph.D. Dissertation, Jadavpur University, Kolkata, 2011.

[5] K. Pretz, "Overcoming Spectrum Scarcity," IEEE Spectrum Newsletter: The Institute Tech Focus, 28 August 2012.

[6] A. Georgakopoulos, K. Tsagkaris, D. Karvounas, P. Vlacheas and P. Demestichas, "Cognitive Radios for Future Internets: Status and Emerging Challenges," IEEE Vehicular Technology Magazine, Vol. 7, No. 3, 2012, pp. 48-56. doi:10.1109/MVT.2012.2204548

[7] F. Tobagi and L. Kleinrock, "Packet Switching in Radio Channels(II) - The Hidden Terminal Problem in Carrier Sense Multiple-Access and Busy-Tone Solution," IEEE Transactions on Communications, Vol. 23, No. 12, 1975, pp. 1417-1433. doi:10.1109/TCOM.1975.1092767

[8] J. So and N. H. Vaidya, "Multi-Channel MAC for AdHoc Networks: Handling Multi-Channel Hidden Terminals Using a Single Transceiver," Proceedings of the 5th ACM International Symposium on Mobile Ad-Hoc Networking and Computing, Tokyo, 24-26 May 2004, pp. 222-233.

[9] I. F. Akyildiz, W. Y. Lee and K. Chowdhury, "CRAHNs: Cognitive Radio Ad-Hoc Networks," Ad-Hoc Networks (Elsevier) Journal, Vol. 7, No. 5, 2009, pp. 810-836.

[10] K. K. Szott, "A Survey of MAC Layer Solutions to Hdden Node Problem in Ad-Hoc Networks," Ad-Hoc Networks, Vol. 10, No. 3, 2012, pp. 635-660. doi:10.1016/j.adhoc.2011.10.003

[11] M. D. Felice, K. R. Chowdhury and L. Bononi, "Model- 
ing and Performance Evaluation of Transmission Control Protocol Over Cognitive Radio Ad-Hoc Networks," Proceedings of the 12th ACM International Conference on Modeling, Analysis and Simulation of Wireless and Mobile Systems, New York, 26-30 October 2009, pp. 4-12.

[12] L. Ma, X. Han and C. C. Shen, "Dynamic Open Spectrum Sharing MAC Protocol for Wireless Ad-Hoc Networks," 2005 1st IEEE International Symposium on New Frontiers in Dynamic Spectrum Access Networks, Baltimore, 8-11 November 2005, pp. 203-213.

[13] K. Xu, M. Gerla and S. Bae, "How Effective Is IEEE 802.11 RTS/CTS Handshake in Ad-Hoc Networks," AdHoc Networks, Vol. 1, No. 1, 2003, pp. 72-76.

[14] S.-L. Wu, C.-Y. Lin, Y. C. Tseng and J. P. Sheu, "A New Multi-Channel MAC Protocol with On-Demand Channel Assignment for Multi-Hop Mobile Ad-Hoc Networks," Proceedings of International Symposium on Parallel Architectures, Algorithms and Networks (I-SPAN), Dallas, 7-9 December 2000, pp. 232-237.

[15] J. Jia, Q. Zhangh and X. Shen, "HC-MAC: Hardware Constrained Cognitive MAC for Efficient Spectrum Management," IEEE Journal on Selected Areas in Communications, Vol. 26, No. 1, 2008, pp. 106-117.

[16] C. Cordeiro and K. Challapali, "C-MAC: A Cognitive MAC Protocol for Multichannel Wireless Networks," Proceedings of IEEE Dynamic Spectrum Access Networks (DySpan), Dublin, 17-20 April 2007, pp. 147-157.

[17] Y. R. Kondareddy and P. Agarwal, "Synchronisation of MAC Protocol for Multi-Hop Cognitive Radio Network," IEEE International Conference on Communications, Beijing, 19-23 May 2008, pp. 3198-3202.

[18] H. Su and X. Zhang, "Cross Layer Based Opportunistic MAC Protocols for QoS Provisioning Over Cognitive Radio Wireless Networks," IEEE Journal on Selected Areas in Communications, Vol. 26, No. 1, 2008, pp. 118-129. doi:10.1109/JSAC.2008.080111

[19] T. Luo, M. Motani and V. Srinivasan, "Cooperative Asynchronous Multi-Channel MAC: Design, Analysis, and Implementation," IEEE Transactions on Mobile Computing, Vol. 8, No. 3, 2009, pp. 338-352. doi:10.1109/TMC.2008.109

[20] P. C. Ng and S. C. Liew, "Throughput Analysis of IEEE802.11 Multi-Hop Ad-Hoc Networks," IEEE/ACM
Transactions on Networking, Vol. 15, No. 2, 2007, pp. 309-322.

[21] Z. H. Velkov and L. Gavrilovska, "Performance of IEEE 802.11 Wireless LANs under Influence of Hidden Terminals and Pareto Distributed Packet Traffic," 1999 IEEE International Conference on Personal Wireless Communication, Jaipur, 17-19 February 1999, pp. 221-225.

[22] J. Li, C. Blake, D. Couto, H. I. Lee and R. Morris, "Capacity of Ad-Hoc Wireless Networks," Proceedings of 7th Annual International Conference on Mobile Computing and Networking, ACM MobiCom, Rome, 16-21 July 2001, pp. 61-69.

[23] R. Tandra and A. Sahai, "SNR Walls for Signal Detection," IEEE Journal of Selected Topics in Signal Processing, Vol. 2, No. 1, 2008, pp. 4-17. doi:10.1109/JSTSP.2007.914879

[24] Y. Zeng and Y.-C. Liang, "Spectrum Sensing Algorithms for Cognitive Radio Based on Statistical Covariance," IEEE Transactions on Vehicular Technology, Vol. 58, No. 4, 2009, pp. 1804-1815. doi:10.1109/TVT.2008.2005267

[25] S. Pattanayak, P. Venkateswaran and R. Nandi, "Artificial Neural Networks for Cognitive Radio," Proceedings of 8th International Conference on Wireless Communication Networking and Mobile Computing, Shanghai, 21-23 September 2012. doi:978-1-61284-683-5/12

[26] J. Mitola, A. Attar, H. O. Harada and H. Aghvami, "Achievements and the Road Ahead: The First Decade of Cognitive Radio," IEEE Transactions on Vehicular Technology, Vol. 59, No. 4, 2010, pp. 1574-1577.

[27] J, Perez-Romero, L. Doyle and M. C. Vuran, "Applications of Cognitive Radio Networks," IEEE Vehicular Technology Magazine, Vol. 7, No. 2, 2012, pp. 23-24. doi:10.1109/MVT.2012.2190226

[28] IEEE 802.11 and 802.11-A Working Group, "Wireless LAN Medium Access Control and Physical Layer (PHY) Specifications: High-Speed Physical Layer in $5 \mathrm{GHz}$ Band," 1999.

[29] D. Vassis and G. Kormentzas, "Throughput Analysis for IEEE 802.11 Ad Hoc Networks under the Hidden Terminal Problem," 3rd IEEE Consumer Communications and Networking Conference, Las Vegas, 8-10 January 2006, pp. 1273-1276. 